\title{
A Heuristic Approach for Blind Source Separation of Instant Mixtures
}

\author{
Jesús Rigoberto Villavicencio Navarro, Luis Márquez Martínez, \\ and Joaquín Álvarez Gallegos \\ CICESE Research Center, \\ Electronics and Telecommunications Department, Ensenada, B.C., \\ Mexico \\ \{jvillavi, Imarquez, jqalvar\}@cicese.mx
}

\begin{abstract}
In this paper we present a methodology for blind source separation (BSS) based on a coherence function to solve the problem of linear instantaneous mixtures of signals. The proposed methodology consists of minimizing the coherence function using a heuristic algorithm based on the simulating annealing method. Also, we derived an analytical expression of the coherence for the BSS model, in which it is found that independent and identically distributed (iid) Gaussian components can be recovered. Our results show satisfactory performance in comparison with traditional methods.
\end{abstract}

Keywords. Blind source separation, second-order statistics, source extraction, Gaussian sources, simulated annealing.

\section{Introduction}

The Blind Source Separation (BSS) problem consists in recovering latent sources from observed mixtures using the statistical information within the data, when information on the sources and mixing process is not known. The BSS problem appears in many multi-sensor systems, ranging from biomedical signal processing [20, $27,28,17,11,28]$ to finance $[19,9]$. The wide set of possible applications makes the BSS problem to acquire great interest.

Among many other interesting applications, an example of the BSS problem considered in this paper is the fetal electrocardiogram (fECG) extraction which is used for diagnostic purposes. This noninvasive technique uses electrodes that pick up maternal ECG (mECG) and, at a lower amplitude, the fECG. The electrodes are also sensitive to other signals, like the skin potentials, electromyograms, and the $50 \mathrm{~Hz}$ interference, which provide a complex signal associated to a mixture of many sources. Due to this, it may be difficult to analyze and obtain a reliable diagnostic. The mixture of the signals is assumed to be instantaneous because it is presumed that the whole body is a heterogeneous conductor in such a way that the recording of the fECG and mECG waveform does not change when the position of the recording electrodes is changed on the surface of the mother's abdomen, only the amplitude of the electrical signal may change [31]. The BSS provides the means to enhance and separate the low amplitude signal coming from the fetal heart from other noises or undesirable signals in a noninvasive way which is useful in early diagnosis of cardiopathologies.

There are two main approaches to solve the BSS problem. In the Independent Component Analysis (ICA) approach [18, 3], separation is based on the statistical distribution of sources. It assumes that sources are possibly independent and identically distributed (iid) but non-Gaussian, and higher order statistics are used as separation criteria to make the recovered components as independent as possible. Another separation scheme is based on Second-Order Statistics (SOS). It assumes that sources are possibly Gaussian but colored. These kinds of sources may show some time structure that can be characterized by a time-delayed covariance matrix which allows identification procedures based on eigen-decomposition [30]. Some other methods use the average eigen-structure by 
taking linear combination of several time-delayed covariance matrices $[1,8]$.

In this paper, we propose the use of a coherence function in order to achieve the recovery of the sources in the instant mixtures problem. Coherence has been considered previously in the BSS problem of convolutive mixtures [15] and in the nonlinear blind source separation problem [26]. In [15], coherence is used as a cost function together with a gradient optimization algorithm to solve the problem of convolutive mixtures. In [26], the coherence function is used to compensate the recovered components distortion. Particle swarm optimization was used for optimization of the cost function which in that case is a weighted sum of mutual information and coherence. Hence, we present an extension of the previous works based on the coherence function in BSS. In this regard, to the best of our knowledge, no work on separation of instant mixtures using the coherence function has been presented up to now.

On the other hand, the BSS problem has no solution for Gaussian and temporally iid sources $[32,2,12]$. As an additional contribution we derived a second algorithm in which source separation of iid Gaussian sources is achieved assuming sources have been mixed by an orthogonal matrix.

This paper is organized as follows. In Section 2 , the problem statements are given along with an analysis of coherence in the BSS model. In Section 3, a cost function based on coherence is derived and implemented in the proposed algorithm. In Section 4, a performance comparison of the proposed algorithm is presented, and a real example of separation of fetal electrocardiogram (fECG) extraction in shown. In Section 5, a discussion of the obtained results is presented, together with some concluding remarks.

\section{Problem Statement}

The BSS model of linear and instantaneous mixtures can be expressed in the following way:

$$
\mathbf{x}(t)=\mathbf{A} \mathbf{s}(t),
$$

where $\mathbf{x}(t) \in \mathbb{R}^{n}$ is the observation vector or sensor signals, $n$ is the number of sensors, $\mathbf{s}(t) \in \mathbb{R}^{m}$ is the vector of unknown sources, and $m$ is the number of sources. In this paper for simplicity we will assume that the number of sensors and sources is the same $(n=m), \quad \mathbf{A} \in$ $\mathbb{R}^{n \times m}$ is a matrix that models the mixing process whose entries are the attenuation that the sources signal suffered from the transmission media, and $t$ is the time index. The BSS problem consists in identification of the mixing matrix $\mathbf{A}$ or its inverse $\mathbf{W}=\mathbf{A}^{-1}$ to estimate the unknown original sources $\mathbf{s}(t)$, using only the information within the finite observed data $x(t)$. The unknown model parameters can be estimated if we assume that the sources are statistically mutually independent or decorrelated. However, neither the scaling of the sources nor their original order can be identified, that is, the separating matrix can be given by $\mathbf{W}=\mathbf{D} \mathbf{P} \mathbf{A}^{-1}$ where $\mathbf{P}$ is a permutation matrix, and $\mathbf{D}$ is a diagonal matrix.

\subsection{Assumptions}

In the analysis presented in this paper we consider the following assumptions unless otherwise stated:

1. $\mathbf{A} \in \mathbb{R}^{n \times m}$ has full column rank $m$.

2. $\mathbf{s}(t)$ is a zero-mean stationary process with nonsingular covariance matrix $\mathbf{R}_{\mathbf{s}}=$ $\mathrm{E}\left\{\mathbf{s}(\mathrm{t}) \mathbf{s}(\mathrm{t})^{\mathrm{T}}\right\}=\operatorname{diag}\left(\sigma_{s_{1}}^{2}, \ldots, \sigma_{s_{m}}^{2}\right)$, where $\sigma_{s_{1}}^{2}$ denotes the variance of the $i$-th source.

The first assumption is related to the mixing model. The columns of the $n \times m$ mixing matrix $\mathbf{A}$ contain the sensor projection weights associated with each source signal. A singular matrix A implies that its determinant is defined and is zero, which applies only for square matrices. If the determinant is zero, it means that the columns of A are dependent, which in its turn means that the sources are completely correlated. The physical meaning of this is that two or more sensors captured exactly the same signal or a linear combination of it. This is probably because the sensors are too close or placed technically at the same spot. The second assumption is related to 
the nature of signals, and it means that they are statistically mutually uncorrelated.

\subsection{Coherence in the Instantaneous BSS Model}

The magnitude-squared coherence (MSC) is a normalized frequency domain function constrained between 0 and 1 for all frequencies. The MSC gives a measure of the linear association between two wide-sense stationary (WSS) zero-mean processes. It has the important property that the coherence between two zeromean uncorrelated processes is zero $[4,5]$. The coherence is given by

$$
\left|\gamma_{x_{1} x_{2}}\right|^{2}=\frac{\left|P_{x_{1} x_{2}}(f)\right|^{2}}{P_{x_{1} x_{1}}(f) P_{x_{2} x_{2}}(f)},
$$

where $P_{x_{1} x_{2}}(f)$ is the cross power spectral density between the processes, $x_{1}(t)$ and $x_{2}(t)$. $P_{x_{1} x_{1}}(f)$ and $P_{x_{2} x_{2}}(f)$ are their respective power spectral densities. Assume the BSS model of linear and instant mixtures for two signals (3):

$$
\begin{aligned}
& x_{1}(t)=a s_{1}(t)+c s_{2}(t), \\
& x_{2}(t)=b s_{1}(t)+d s_{2}(t) .
\end{aligned}
$$

Assume $s_{1}(t)$ and $s_{2}(t)$ are uncorrelated with zero-mean. Then,

$$
P_{x_{1} x_{1}}(f)=a^{2} P_{S_{1} s_{1}}(f)+c^{2} P_{s_{2} s_{2}}(f)
$$

and

$$
P_{x_{2} x_{2}}(f)=b^{2} P_{s_{1} s_{1}}(f)+d^{2} P_{s_{2} s_{2}}(f) \text {. }
$$

The cross power spectral density between $x_{1}(t)$ and $x_{2}(t)$ is

$$
P_{x_{1} x_{2}}(f)=a b P_{s_{1} s_{1}}(f)+c d P_{s_{2} s_{2}}(f) .
$$

Substituting (4), (5) and (6) in (2) yields (7); the coherence between the observations in the instantaneous BSS model:

$$
=\frac{\left|\gamma_{x_{1} x_{2}}(f)\right|^{2}}{[a b)^{2} P_{S_{1} s_{1}}^{2}(f)+(c d)^{2} P_{S_{2} s_{2}}^{2}(f)}=
$$

If the mixing matrix is a diagonal matrix (i.e., $b=c=0$ ), the observation vector will consist of the unmixed source signals. Substituting these parameters' values in (7) we can find out that $\left|\gamma_{x_{1} x_{2}}\right|^{2}=0$. The coherence is zero for uncorrelated processes. We may note that, in the observation vector, the matrix A will differ from a diagonal matrix. However, by applying a whitening transformation $\mathbf{V}$ and an orthogonal rotation transformation $\mathbf{R}$ to the observed data, the resulting matrix (global matrix) is diagonal.

\subsection{Coherence of Mixed Gaussian Sources with iid Time Structure}

The power spectral density of two zero-mean Gaussian stationary sources with iid time structure and unit variance will have the same shape, that is, $P_{s_{1} s_{1}}(f)=P_{s_{2} s_{2}}(f)$. If the observed data is a mixture of them, Eq. (7) can be reduced to the following expression:

$$
\left|\gamma_{x_{1} x_{2}}\right|^{2}=\frac{(a b)^{2}+(c d)^{2}}{\left[a^{2}+c^{2}\right]\left[b^{2}+d^{2}\right]} .
$$

In this case, as shown by Carter (1993), the MSC becomes frequency independent, and in consequence, the estimate of the coherence is no longer dependent on the time structure of the sources. In this way, it is observed that the coherence estimate in (8) will be dependent on the variance ratio between sources provided by the mixing matrix. From (8) it is found that the variance difference between sources mixture adds information that can be used to achieve separation, thus the problem becomes a variance maximization problem. An example is shown in Figure 4 for two orthogonally mixed Gaussian iid sources. Every curve is generated from the coherence estimate using a specific number of averaged segments, as indicated in the key. Assuming that the Gaussian iid sources have been mixed by an orthogonal matrix, the coherence estimate will be minimum, when the recovered components are independent. As the variance ratio between the sources increases, the separation performance of the Gaussian iid signals increases, see Figure 5.

In the case where the observation vector $\mathbf{x}$ is whitened, for any rotation $\theta$ the coherence 
estimate in (8) will remain constant. The whitening transform would destroy the variance information and must be avoided in this case. The limitation of the identifiability condition [30] of SOS methods is verified as well; if the sources' power spectral densities are not linearly independent, they cannot be estimated.

\section{Proposed Method}

\subsection{Pre-Processing}

In BSS it is a common procedure to apply some pre-processing to the observed data in order to simplify the problem. The first step is to remove the mean from the observation. The second step is to perform a pre-whitening transformation which is based on eigen-value decomposition [19]:

$$
\mathbf{y}(t)=\mathbf{V x}(t),
$$

where $\quad \mathbf{V}=\mathbf{D}^{-1 / 2} \mathbf{E}^{T}, \mathbf{E}=\left(e_{1} \ldots e_{m}\right)$ is the orthogonal matrix of the eigenvector of the covariance matrix $\mathrm{C}_{\mathrm{x}}=\mathrm{E}\left\{\mathrm{xx}^{\mathrm{T}}\right\}$, and $\mathrm{D}=$ $\operatorname{diag}\left(\mathrm{d}_{1} \ldots \mathrm{d}_{\mathrm{m}}\right)$ is a diagonal matrix formed by the eigenvalues of $C_{x}$. The whitened components will have the statistical properties of being uncorrelated, and their variances will be equal to unity [19]. This pre-processing reduces the source separation problem of finding an orthogonal (rotation) factor $\mathrm{R}$, where $\mathrm{RV}=\mathrm{W}$.

\subsection{MSC Criterion for BSS}

The orthogonal rotation matrix $\mathrm{R}$ is composed of a product of successive Givens rotations $R\left(\theta_{i, j}\right) \in$ $\mathbb{R}^{m \times m}$, defined for $i<j$ by

$$
R\left(\theta_{i, j}\right)=\left[\begin{array}{ccccccc}
1 & 0 & \cdots & \cdots & \cdots & \cdots & 0 \\
0 & \ddots & \vdots & & \vdots & & \vdots \\
\vdots & \cdots & c & \cdots & s & \cdots & 0 \\
\vdots & & \vdots & \ddots & \vdots & & \vdots \\
\vdots & \cdots & -s & \cdots & c & \cdots & 0 \\
\vdots & & \vdots & & \vdots & \ddots & \vdots \\
0 & \cdots & \cdots & \cdots & \cdots & \cdots & 1
\end{array}\right]
$$

where $c=\cos \left(\theta_{i, j}\right)$ and $s=\sin \left(\theta_{i, j}\right)$ appear at the intersection of the $\mathrm{i}$-th and j-th rows and columns. This represents a clockwise rotation of $\theta_{i, j}$ radians in the $(i, j)$ plane. For every pair $(i, j)$, under the restriction $1<i<j<m$, there exists a rotation angle. Thus, the number of rotation angles is $M=m(m-1) / 2$. The new estimated components a given by

$$
\begin{gathered}
\hat{\mathbf{s}}(\theta)=\mathbf{R}(\theta) \mathbf{V} \mathbf{x}(t), \\
\hat{\mathbf{s}}\left(\theta_{1}, \theta_{2}, \ldots, \theta_{M}\right)=R\left(\theta_{1}\right) R\left(\theta_{2}\right) \ldots R\left(\theta_{M}\right) \mathbf{V} \mathbf{x}(t),
\end{gathered}
$$

where $\theta=\left[\theta_{1}, \theta_{2}, \ldots, \theta_{M}\right]$, and

$$
\mathbf{R}(\theta)=R\left(\theta_{1}\right) R\left(\theta_{2}\right) \ldots R\left(\theta_{M}\right) .
$$

The method consists in applying Givens rotations until the cost function had been minimized.

A cost function for more than two sources can be derived if we use the sum of the MSC between all $m(m-1) / 2$ pairs of vectors $(l, k)$ of $\hat{\mathbf{s}}$, as shown in [15]. We can express $\Gamma_{\hat{s}}$ as the matrix of sum of coherence functions between all pairs of vectors of $\hat{\mathbf{s}}$ :

$$
\Gamma_{\hat{\mathbf{s}}}=\left[\begin{array}{cccc}
\sum\left|\gamma_{\hat{s}_{1} \hat{s}_{1}}(f)\right|^{2} & \sum\left|\gamma_{\hat{s}_{1} \hat{s}_{2}}(f)\right|^{2} & \cdots & \sum\left|\gamma_{\hat{s}_{1} \hat{s}_{m}}(f)\right|^{2} \\
\sum\left|\gamma_{\hat{s}_{2} \hat{s}_{1}}(f)\right|^{2} & \sum\left|\gamma_{\hat{S}_{2} \hat{s}_{2}}(f)\right|^{2} & \vdots & \vdots \\
\vdots & \vdots & \ddots & \vdots \\
\sum\left|\gamma_{\hat{s}_{m} \hat{s}_{1}}(f)\right|^{2} & \cdots & \cdots & \sum\left|\gamma_{\hat{s}_{m} \hat{s}_{m}}(f)\right|^{2}
\end{array}\right] .
$$

Since $\quad \sum\left|\gamma_{\hat{s}_{k} \hat{s}_{l}}(f)\right|^{2}=\sum\left|\gamma_{\hat{s}_{l} \hat{s}_{k}}(f)\right|^{2}, \quad$ and $\sum\left|\gamma_{\hat{s}_{l} \hat{s}_{k}}(f)\right|^{2}=1$ for all $f$ when $l=k, \Gamma_{\hat{\mathbf{s}}}$ is a symmetric matrix. When the vectors of $\hat{\mathbf{s}}$ are mutually uncorrelated, $\Gamma_{\hat{s}}$ is a diagonal matrix. Then, is desirable to minimize the upper triangle terms of $\Gamma_{\hat{s}}$. In this way, only the upper triangle terms of (14) are considered in the cost function (15).

$$
\mathbf{J}=\sum_{l, k} \sum\left|\gamma_{\hat{s}_{l} \hat{s}_{k}}(f)\right|^{2}
$$

where $1<l<k<M$.

Metaheuristics are widely implemented in multivariable problems [12, 24]. We implemented 
Table 1. Montecarlo results for colored Gaussian sources; $\mu$ and $\sigma$ are the SIR mean and standard deviation for 50 tests, respectively; $n_{d}$ is the number of averaged segments used in the estimation of coherence

\begin{tabular}{cccccc}
\hline Algorithm & & \multicolumn{4}{c}{ Colored Gaussian sources $\operatorname{SIR}_{\mathrm{dB}}$} \\
& & & $\left(\mathrm{s}_{1}, \mathrm{~s}_{3}\right)$ & \multicolumn{2}{c}{$\left(\mathrm{s}_{1}, \mathrm{~s}_{2}, \mathrm{~s}_{3}\right)$} \\
\hline & $\mathrm{n}_{\mathrm{d}}$ & $\mu$ & $\sigma \mathrm{e}-9$ & 41.01 & $\sigma$ \\
SOBI & & 48.90 & 5.53 & 36.13 & 11.78 \\
MSC-BSS & 8 & 71.91 & 4.58 & 39.60 & 10.95 \\
MSC-BSS & 16 & 71.84 & 3.73 & 39.04 & 10.12 \\
MSC-BSS & 32 & 71.28 & & & \\
\hline
\end{tabular}

the Simulated Annealing ${ }^{1}$ (SA) [22] method to find $\theta$, the minimizer of $\mathbf{J}$ in the range $[0<\theta<\pi / 2]$. A fast temperature dropping schedule is used to improve the separation performance. The basic steps to achieve separation of independent components are shown in Algorithm 1.

Algorithm 1: MSC-BSS algorithm

1. Remove the mean from the observations.

2. Perform a pre-whitening transformation by Eq. (9).

3. Get an initial solution $\theta$ with energy J:

Pick a random initial solution $\theta$.

Generate a rotation matrix by Eq. (13), $R(\theta)$.

Generate the estimated sources by Eq. (12).

Calculate the cost function $\mathbf{J}$.

4. Select an initial "high temperature", $T$.

5. while the maximum number of iterations or minimum temperature is not reached do

\section{begin}

Pick a random "nearby" solution $\theta_{p}$.

Generate a rotation matrix by

\footnotetext{
1 Matlab's Optimization toolbox parameters (simulannealbnd): TemperatureFcn: @temperaturefast; Maxlter: $m^{*} 300$;

InitialTemperature: 0.1; Reanneallnterval: 400.
}

Eq. (13), $R\left(\theta_{p}\right)$.

Generate the estimated sources by Eq. (12).

Calculate the cost function $\mathbf{J}_{p}$.

Let $\Delta \mathbf{J}=\mathbf{J}_{p}-\mathbf{J}$

If $\Delta \mathbf{J} \leq \mathbf{0}$

$$
\theta \leftarrow \theta_{p}
$$

else

$$
\begin{aligned}
& \theta \leftarrow \theta_{p} \text { with an } \\
& \text { acceptance probability }{ }^{2} \\
& \text { else }
\end{aligned}
$$

Reject solution, $(\theta \leftarrow \theta)$

end

Reduce temperature $T$ by $\Delta T$

\section{end while}

6. $\theta$ is the best solution found by the SA in the range $[0, \pi / 2]$.

7. Estimate the original sources by Eq. (12).

\section{Experiments and Results}

The MSC-BSS method was implemented in the ICALAB toolbox [7] and compared to other SOS methods (SOBI [1]). The signal-to-interference-

\footnotetext{
2 @acceptancesa: Matlab's simulated annealing acceptance function.
}

Computación y Sistemas, Vol. 18, No. 4, 2014, pp. 719-730 doi: 10.13053/CyS-18-4-1951 
ratio (SIR) [6] index was used. The coherence function is calculated via the Welch method with $50 \%$ of overlap between segments. A Hamming window is applied to every segment before averaging.

\subsection{Separation of Colored Gaussian Sources}

We consider the separation of colored Gaussian sources for mixtures of two and three sources. Three sources, $s_{1}, s_{2}$, and $s_{3}$, were used for the experiments. They were obtained by low-pass filtering white Gaussian noise through a fourth order Butterworth filter with cut frequency of 0.4 , 0.7 and 0.9 , respectively. The mixing matrix $\mathbf{A}$ was generated randomly. Algorithm 1 was implemented with different number of averaged segments $n_{d}=8,16,32$ in the estimation of the coherence.

Table 1 shows the comparison results for 50 tests. From the results we see that the MSC method performance degrades as the number of sources to retrieve increases. Also, a reduction of the SIR's standard deviation with an increasing number of averaged segments is observed.

\subsection{Separation of Colored Non-Stationary Sources}

In this section an example of mixed lung and cardiac sounds is considered. The purpose of this test is to verify the algorithm performance when non-stationary signals are used, although it may not reflect a real sources environment. The observations were generated with a heart sound and a respiratory sound taken from [10, 23], respectively. Each signal consisted of $60 \mathrm{k}$ samples sampled at a frequency of $8000 \mathrm{~Hz}$. Figure 1 shows the time series and power spectral density of the sources.

In Table 2 we show the results of a Montecarlo simulation of 100 tests. In each test, the mixing matrix A was generated randomly (uniform distribution). The matrix $\mathbf{W}$ was estimated using

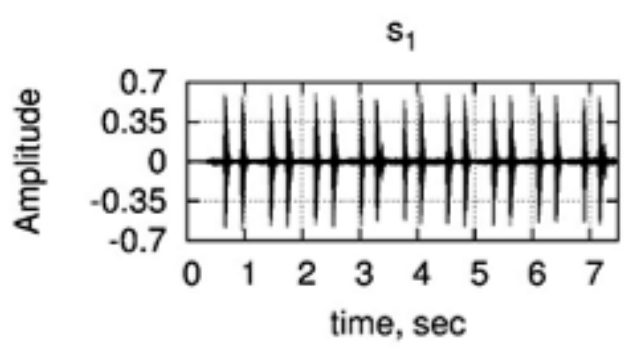

(a)

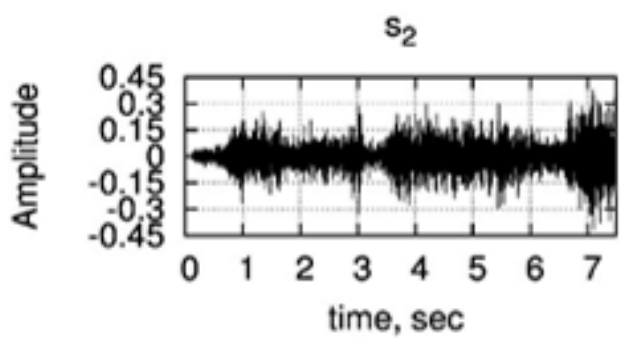

(c)

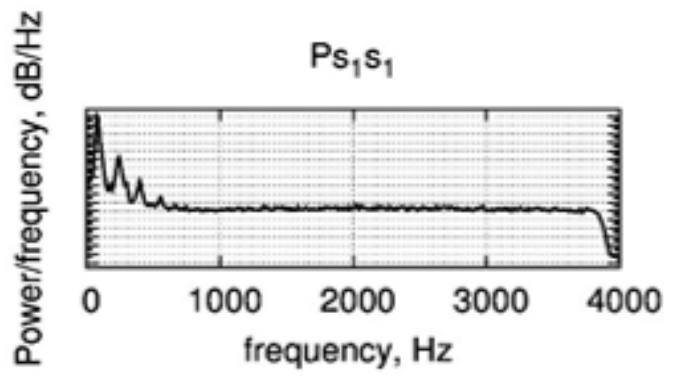

(b)

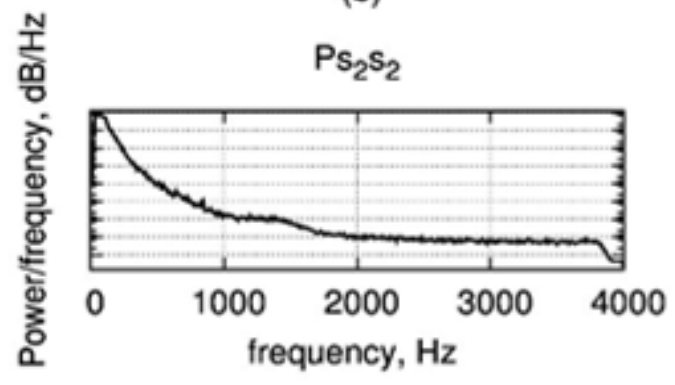

(d)

Fig. 1. (a) Heart sound $s_{1}(t)$, (b) $P_{s_{1} s_{1}}$ heart sound power spectral density, (c) lung sound $s_{2}(t)$, (d) $P_{S_{2} S_{2}}$ lung sound power spectral density 
Table 2. Montecarlo test results using non-stationary sources; $\mu$ and $\sigma$ are the respective SIR mean and standard deviation, $n_{d}$ is the number of segments used in the estimation of coherence

\begin{tabular}{cccc}
\hline \multicolumn{2}{c}{ Algorithm } & \multicolumn{2}{c}{ Non-stationary sources $\boldsymbol{S I \boldsymbol { R } _ { \boldsymbol { d } \boldsymbol { B } }}$} \\
\hline & $n_{d}$ & $\mu$ & $\sigma$ \\
\hline SOBI & & 6.96 & 5.18 \\
$\begin{array}{c}\text { MSC- } \\
\text { BSS }\end{array}$ & 8 & 37.63 & 0.0131 \\
$\begin{array}{c}\text { MSC- } \\
\text { BSS }\end{array}$ & 16 & 38.31 & 0.0174 \\
$\begin{array}{c}\text { MSC- } \\
\text { BSS }\end{array}$ & 32 & 38.54 & 0.0180 \\
\hline
\end{tabular}

Algorithm 1, and the separation performance was obtained using the SIR performance index. The performance of the MSC-BSS algorithm is satisfactory regardless the sources are nonstationary.

\subsection{An Example of Real fECG Extraction}

In this section we show the separation or enhancement of real fECG data by means of the proposed Algorithm 1. The data was obtained from the Abdominal and Direct Fetal Electrocardiogram Database which contains multichannel fetal electrocardiogram (FECG) recordings [21]. Each recording comprises four differential signals acquired from maternal abdomen and the reference direct fetal electrocardiogram registered from the fetal head. Additional information from [16]: signals recorded in labor, between 38 and 41 weeks of gestation; four signals acquired from maternal abdomen; direct electrocardiogram recorded simultaneously from fetal head; positioning of electrodes was constant during all recordings; $\mathrm{Ag}-\mathrm{AgCl}$ electrodes (3M Red Dot 2271) and abrasive material to improve skin conductance (3M Red Dot Trace Prep 2236); Bandwidth: $1 \mathrm{~Hz}-150 \mathrm{~Hz}$ (synchronous sampling of all signals); additional digital filtering for removal of power-line interference $(50 \mathrm{~Hz})$ and baseline drift; sampling rate: $1 \mathrm{kHz}$; resolution: 16 bits.
Figure 2 shows 5000 samples of the four channels recorded data. Each sensor provides a complex signal in which the fECG and mECG are contained along with other undesirable signals. Figure 3 shows the output of the proposed Algorithm 1. The direct fetal electrocardiogram is shown for purposes of comparison with the extracted fECG signal. The estimated source 3 clearly shows the enhanced and denoised $\mathrm{mECG}$. The estimated sources 1 and 2 show some background noise or undesired signals. The estimated source 4 shows the enhanced fECG but is still mixed with the mECG, due to this a further post-processing is conducted as shown in [31]. However, the BSS represents a very important first step in the enhancement of ECG signals.

\subsection{A Case of Separation of Mixed Gaussian Processes with iid Time Structure}

In this section we deal with the separation of Gaussian iid sources. The assumptions are the following:

1. A is of full column rank $m$ and orthogonal.

2. The source signals $\mathbf{s}(t)$ are statistically mutually independent iid Gaussian sources.

As found in (8), for mixed Gaussian sources with iid time structure, the coherence estimate depends only on the power of the observed mixtures (variance maximization). Hence, the variance ratio between the sources can be used to find a transformation where the coherence is minimal. In Figure 2, an example is shown for two orthogonally mixed iid Gaussian WSS processes with a variance ratio of $\sigma_{i} / \sigma_{j}=4, A=\left[\begin{array}{ll}2 & 0 \\ 0 & 1\end{array}\right]$. Figure 2 is obtained by applying a rotation transformation $\mathbf{R}$ to the observed mixtures from $[-\pi / 4, \pi / 4]$. The minimum of the function $\mathbf{J}$ occurs when $\mathbf{R}=\mathbf{I}$. It is worth noting that the minimum of $\mathbf{J}$ is not affected by the bias in the coherence estimate. Nonetheless, the bias is reduced with the increasing number of averaged segments, as we see, the minimum reaching zero. Algorithm 2 is similar to Algorithm 1 except that the prewhitening step is omitted. 

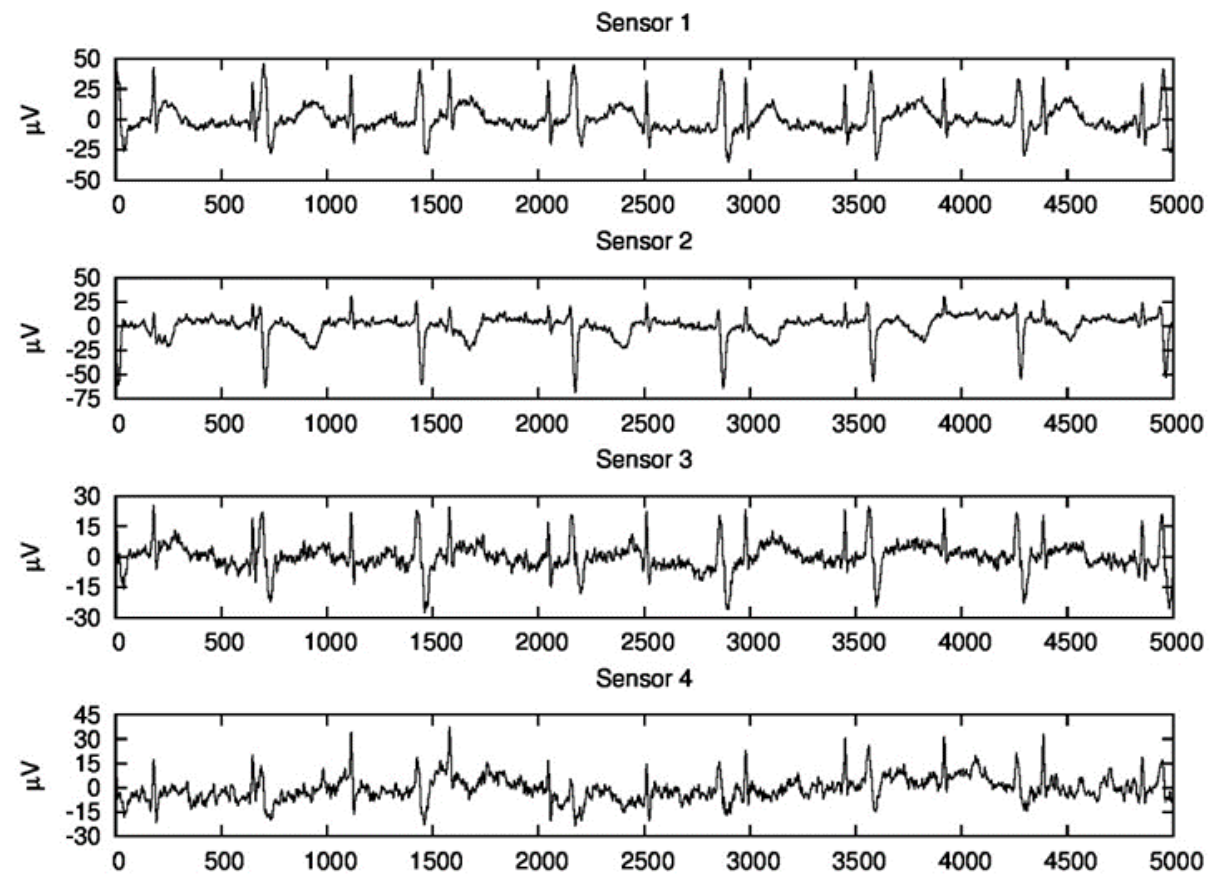

Fig. 2. Four channels mother ECG abdominal recording

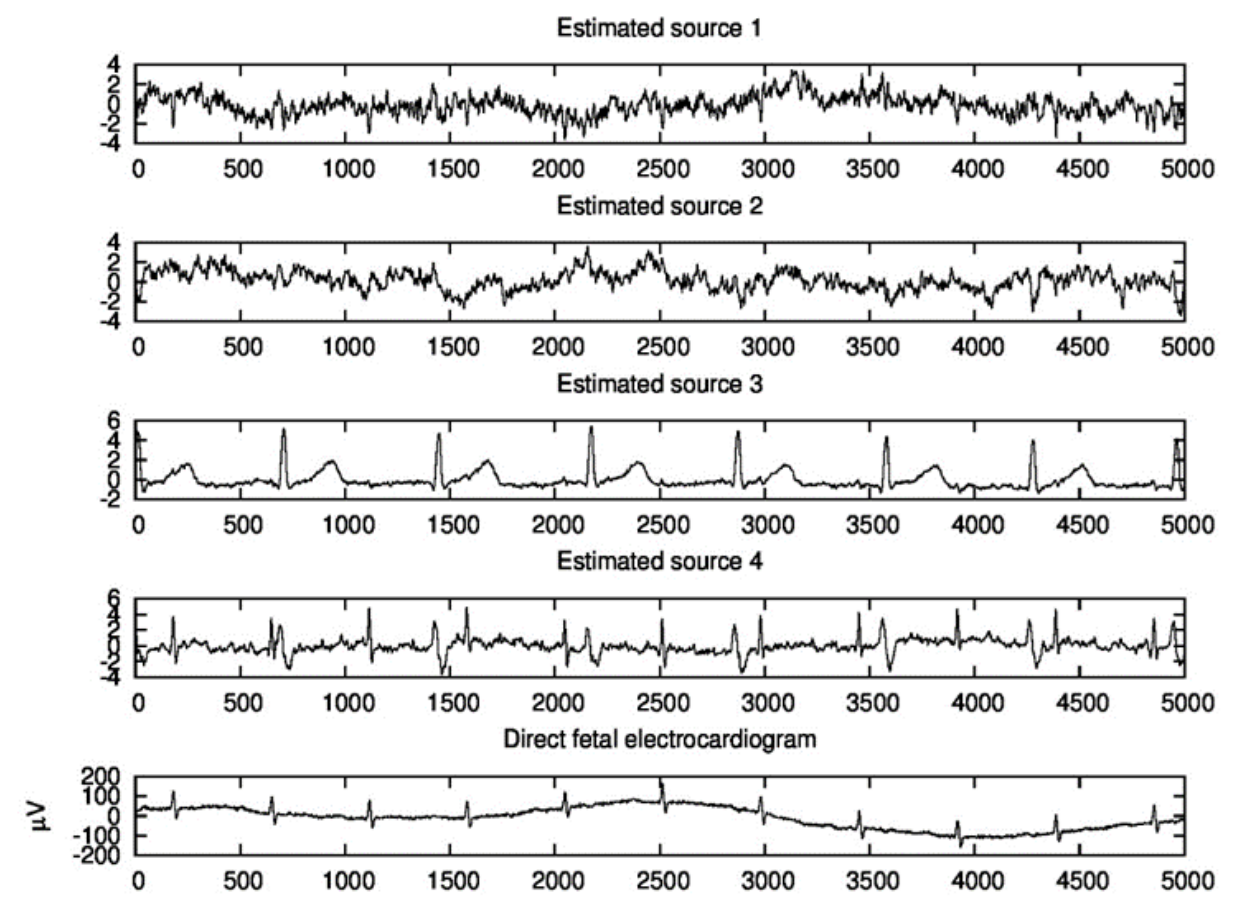

Fig. 3. Estimated sources with the proposed Algorithm 1 and a direct fetal electrocardiogram recorded from the fetus head 
Algorithm 2: MSC-BSSiid algorithm

1. Remove the mean from the observations.

2. Get an initial solution $\theta$ with energy $\mathrm{J}$ :

Pick a random initial solution $\theta$.

Generate a rotation matrix by Eq. (13), $R(\theta)$.

Generate the estimated sources by $\hat{\boldsymbol{s}}=\mathbf{R x}(t)$.

Calculate the cost function $\mathbf{J}$.

3. Select an initial "high temperature", $T$.

4. while the maximum number of iterations or minimum temperature is not reached do

\section{begin}

Pick a random "nearby" solution $\theta_{p}$.

Generate a rotation matrix by Eq. (13), $R\left(\theta_{p}\right)$.

Generate the estimated sources by $\hat{\boldsymbol{s}}=\mathbf{R} \mathbf{x}(t)$

Calculate the cost function $\mathbf{J}_{p}$.

Let $\Delta \mathbf{J}=\mathbf{J}_{p}-\mathbf{J}$

If $\Delta \mathbf{J} \leq \mathbf{0}$

$$
\theta \leftarrow \theta_{p}
$$

else

$$
\begin{aligned}
& \theta \leftarrow \theta_{p} \text { with an } \\
& \text { acceptance probability }{ }^{3} \\
& \text { else }
\end{aligned}
$$

Reject solution $(\theta \leftarrow \theta)$

\section{end}

Reduce temperature $T$ by $\Delta T$

\section{end while}

5. $\theta$ is the best solution found by the SA in the range $[0, \pi / 2]$.

6. Estimate the original sources by $\hat{\boldsymbol{s}}=\mathbf{R x}(t)$.

3 @acceptancesa: Matlab's simulated annealing acceptance function.

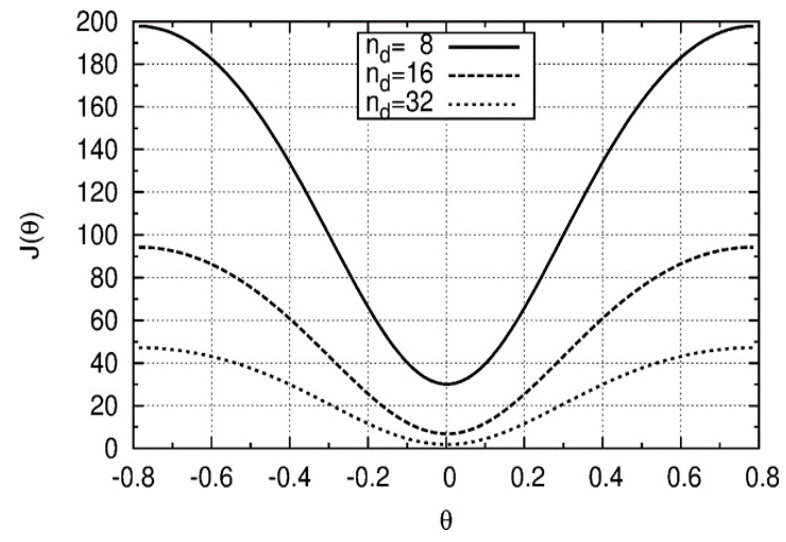

Fig. 4. The cost function $J$ for two orthogonally mixed Gaussian iid sources. Every curve is generated from the coherence estimate using a specific number of averaged segments as indicated in the key. The variance ratio between the sources is $\sigma_{i} / \sigma_{j}=4$

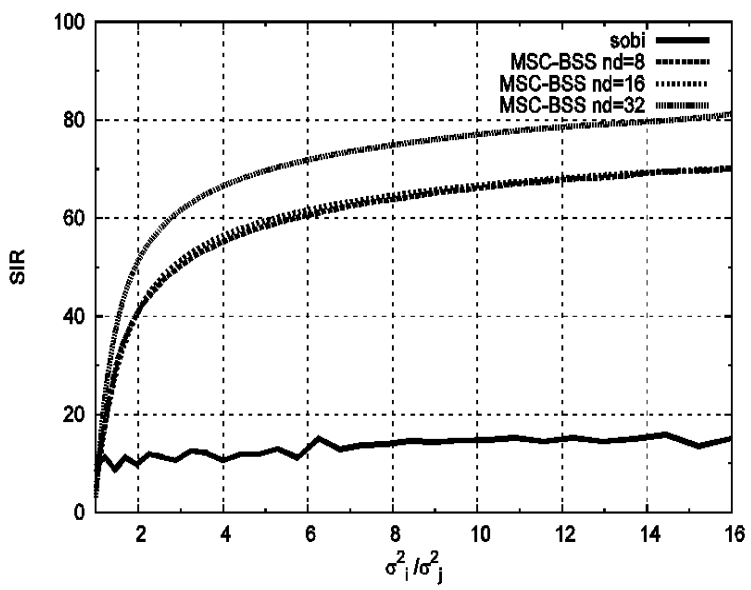

Fig. 5. SIR vs variance ratio sources. Results of separation performance for Gaussian sources with iid time structure; $2^{16}$ samples were used in the test, $i=1$, $j=2$

We consider a comparison of the extraction of the Gaussian iid sources with an increasing variance ratio. Two iid Gaussian WSS processes with zero mean were used for the test, $s_{1}(t)$ and $s_{2}(t)$, where $P_{s_{1} s_{1}}=\sigma_{1}^{2}$ and $P_{s_{2} s_{2}}=\sigma_{2}^{2}=1 . \sigma_{1}^{2}$ was chosen accordingly to change the variance ratio from 1 to $16 ; 50$ tests were performed per variance ratio. The observation vector was 
generated by the generative model $\hat{\mathbf{s}}=\mathbf{R}(\theta) \mathbf{s}(\mathrm{t})$ where $\mathbf{R}(\theta)$ is a rotation matrix, Eq. (13), and $\theta$ is the rotation angle which was chosen randomly. The MSC-BSS and SOBI algorithm were used to estimate the matrix $\mathbf{W}$ from the observations; the whitening step was not performed in this test.

The separation performance of the Gaussian sources with iid time structure improves with an increasing ratio between the variances of the original signals. For a 2:1 ratio good separation is achieved. This can be seen in Figure 5, where a plot of the average SIR estimate for several variance ratio between the sources is shown. Unexpectedly, the SOBI algorithm performance improved gradually as the ratio of the variances between the signals increased. However, its performance is not comparable with the one obtained by the MSC-BSS algorithm.

\section{Conclusions}

In this paper we present a methodology for the BSS based on the coherence function to solve the problem of instantaneous linear mixtures. The proposed methodology seeks to minimize the cost function based on coherence by a heuristic method, simulated annealing. The results showed a satisfactory performance in comparison with a state of art method. In addition, we also present a special case where, modifying our methodology, iid Gaussian sources can be separated by variance maximization. This represents an advantage over other methods because those cannot treat this special case.

\section{References}

1. Belouchrani, A., Abed-Meraim, K., Cardoso, J.F. \& Moulines, E. (1997). A blind source separation technique using second-order statistics, IEEE Transactions on Signal Processing, Vol. 45, No. 2: pp. 434-444.

2. Cardoso, J.F., Snoussi, H., Delabrouille, J. \& Patanchon, G. (2002). Blind separation of noisy Gaussian stationary sources. Application to cosmic microwave background imaging. ArXiv Astrophysics e-prints.

3. Cardoso, J., \& Souloumiac, A. (1993). Blind beamforming for non-gaussian signals. Radar and
Signal Processing, IEE Proceedings F, Vol. 140, No. 6, pp. 362-370.

4. Carter, G.C. (1972). Estimation of the magnitudesquared coherence function (spectrum). Tech. Rep. AD0743945, Naval underwater systems center Newport RI, New London, Conn.

5. Carter, G.C. (1993). Coherence and time delay estimation an applied tutorial for research, development, test, and evaluation engineers. IEEE Press.

6. Cichocki, A., \& Amari, S. (2003). Adaptive Blind Signal and Image Processing. John Wiley and Sons, Ltd.

7. Cichocki, A., Amari, S., Siwek, K., Tanaka, T. \& Anh Huy Phan. ICALAB Toolboxes [online].

8. Choi, S., \& Cichocki, A. (2000). Blind separation of nonstationary sources in noisy mixtures. Electronics Letters, Vol. 36, No. 9, pp. 848-849.

9. Comon, P. \& Jutten, C. (2010). Handbook of Blind Source Separation: Independent Component Analysis and Applications. Academic Press.

10. Corporation, S. (2002), Auscultation skills: breath \& heart sounds. Springhouse.

11. Crespo-Garcia, M., Atienza, M., \& Cantero, J.L. (2008). Muscle artifact removal from human sleep EEG by using independent component analysis. Annals of biomedical engineering, Vol. 36, No. 3, pp. 467-475.

12. Darmois, G. (1953). Analyse générale des liaisons stochastiques: etude particulière de l'analyse factorielle linéaire. Revue de l'Institut International de Statistique / Review of the International Statistical Institute, Vol. 21, No. 1/2, pp. 2-8.

13. Díaz Pando, H., Cuenca Asensi, S., Sepúlveda Lima, R., Fajardo Calderín, J., \& Rosete Suárez, A. (2013). Aplicación de lógica difusa para el particionado hardware/software en sistemas embebidos. Computación y Sistemas, Vol. 17, No. 1, pp. 25-39.

14. Duarte, L., Rivet, B., \& Jutten, C. (2010). Blind extraction of smooth signals based on a secondorder frequency identification algorithm. Signal Processing Letters IEEE, Vol. 17, No. 1, pp. 79-82

15. Fancourt, C. \& Parra, L. (2001). The coherence function in blind source separation of convolutive mixtures of non-stationary signals. Neural Networks for Signal Processing XI, Proceedings of the IEEE Signal Processing Society Workshop, pp. 303-312.

16. Goldberger, A.L., Amaral, L.A.N., Glass, L., Hausdorff, J.M., Ivanov, P.Ch., Mark, R.G., Mietus, J.E., Moody, G.B., Peng, C.-K., Stanley, H.E. (2000). PhysioBank, PhysioToolkit, and PhysioNet, Components of a New Research 
Resource for Complex Physiologic Signals. Circulation, Vol. 101, No. 23, pp. e215e220.

17. Hesse, C.W. (2008). Model order estimation for blind source separation of multichannel magnetoencephalogram and electroencephalogram signals. Engineering in Medicine and Biology Society, EMBS 2008, 30th Annual International Conference of the IEEE, pp.3348-3351, IEEE.

18. Hyvärinen, A. (1999). Fast and robust fixed-point algorithms for independent component analysis. IEEE Transactions on Neural Networks, Vol. 10, No. 3, pp. 626-634.

19. Hyvärinen, A. \& Oja, E. (2000). Independent component analysis: algorithms and applications. Neural Networks, Vol. 13, No. 4-5, pp. 411-430.

20. James, C.J. (2004). Introduction and overview of the BSS/ICA problem-specifically when applied to biomedicine. IEEE Seminar on Blind Source Separation in Biomedicine.

21. Jezewski, J., Matonia, A., Kupka, T., Roj, D. \& Czabanski, R. (2012). Determination of the fetal heart rate from abdominal signals: evaluation of beat-to-beat accuracy in relation to the direct fetal electrocardiogram. Biomedical Engineering/ Biomedizinische Technik, Vol. 57, No. 5, pp. 383394.

22. Kirkpatrick, S., C.D. Gelatt, \& M.P. Vecchi (1983). Optimization by simulated annealing. Science, Vol. 220, No. 4598, pp. 671-680.

23. Lehrer, S. (2002). Understanding lung sounds. W.B. Saunders.

24. Machin Navas, M., \& Nebro Urbaneja, A.J. (2013). Metaheurísticas multiobjetivo adaptativas. Computación y Sistemas, Vol. 17, No. 1, pp. 5362.

25. Moudden, Y., Cardoso, J.F., Starck, J.L. \& Delabrouille, J. (2005). Blind component separation in wavelet space: application to $\mathrm{cmb}$ analysis. EURASIP J. Appl. Signal Process., Vol. 2005, pp. 2437-2454.

26. Oku,T. \& Sano, A. (2003). Nonlinear blind source separation using coherence function. SICE 2003 Annual Conference, pp. 2550-2560

27. Pourazad, M.T., Moussavi, Z., Farahmand, F. \& Ward, R.K. (2005). Heart Sounds Separation From Lung Sounds Using Independent Component Analysis. Engineering in Medicine and Biology Society, 27th Annual International Conference of the IEEE-EMBS, pp. 2736-2739.

28. Romero, S., Mañanas, M.A., \& Barbanoj, M.J. (2008). A comparative study of automatic techniques for ocular artifact reduction in spontaneous EEG signals based on clinical target variables: a simulation case. Computers in biology and medicine, Vol. 38, No. 3, pp. 348-360.

29. Romo-Vázquez, R., Vélez-Pérez, H., Ranta, R., Louis Dorr, V., Maquin, D., \& Maillard, L. (2012). Blind source separation, wavelet denoising and discriminant analysis for eeg artefacts and noise cancelling. Biomedical Signal Processing and Control, Vol. 7, No. 4, pp. 389-400.

30. Tong, L., Liu, R.W., Soon, V., \& Huang, Y.F. (1991). Indeterminacy and identifiability of blind identification. IEEE Transactions on Circuits and Systems, Vol. 38, No. 5, pp. 499-509.

31. Vigneron, V., Paraschiv-lonescu, A., Azancot, A., Sibony, O., \& Jutten, C. (2003). Fetal electrocardiogram extraction based on nonstationary ICA and wavelet denoising. Proceedings. Seventh International Symposium on Signal Processing and Its Applications, IEEE, Vol. 2, pp. 69-72.

32. Yeredor, A. (2012). Multiple-snapshots bss with general covariance structures: A partial maximum likelihood approach involving weighted joint diagonalization. Signal Processing, Vol. 92, No. 8, pp. 1832-1843

Jesús Rigoberto Villavicencio Navarro obtained a degree in Electronics Engineering from Universidad Autónoma de Baja California (Mexico) in 2001. In 2005 received his M.Sc. degree from the Scientific Research and Advanced Studies Center of Ensenada, Mexico (CICESE). At present he is a PhD. student specialized in Control and Instrumentation at CICESE. His research interests include digital signal processing and nonlinear control.

Luis Alejandro Márquez Martínez received his B.S. in Electronics Engineering from the Universidad Autónoma de Baja California, Mexico, in 1992, the M.S. from the CICESE Research Center, at Ensenada B.C., Mexico, in 1994, and the Ph.D. from the Ecole Centrale de Nantes and the Université de Nantes, France, in 2000. He held a post-doctoral position at the University of Pretoria in 2000. He is with the CICESE Research Center since 2001. He has been a visiting professor at the UTIA (Czech Republic), and the Ecole Centrale de Nantes (France). His research interests include algebraic and geometric methods for nonlinear time-delay 
systems, optimization, symbolic algebra, signal processing, and instrumentation.

Joaquin Álvarez Gallegos obtained the Doctor degree in Automatic Control from the National Polytechnique Institute of Grenoble, France (1979). At present he is a full time professor at the Electronics and Telecommunications Department of the Scientific Research and Advanced Studies Center of Ensenada, Mexico (CICESE). Prof. Alvarez has been a member of the National
System of Researchers of Mexico since 1984, level III at present. Also, he is a member of the Mexican Academy of Sciences, the Mexican Academy of Engineering, the IEEE, and the Mexican Association of Automatic Control. His research interests are in the fields of nonlinear control, discontinuous systems, chaos control and synchronization.

Article received on 13/03/2014, accepted on 08/05/2014. 